\title{
C-01
}

\section{ANÁLISIS DEL FUNCIONAMIENTO DE UNA RED COLECTIVA ANTE DISTINTAS ESTRATEGIAS DE RIEGO EN PARCELA}

\author{
Lima, F.A. ${ }^{1}$, Córcoles, J.I ${ }^{2}$, Moreno, M.A ${ }^{4}$,Tarjuelo, J.M $M^{5}$ Martínez-Romero, A.M ${ }^{3}$
}

${ }^{1}$ Doctorando, Universidad Castilla-La Mancha (UCLM), Centro Regional de Estudios del Agua, aldiel_metal@hotmail.com

${ }^{2}$ Dr. Ingeniero Agrónomo, Profesor Contratado Doctor Temporal EIIAB, Departamento de Mecánica Aplicada e Ingeniera de Proyectos, UCLM, juanignacio.corcoles@uclm.es

${ }^{3}$ Dr. Ingeniero Agrónomo, Profesor Asociado; EIIAB, Departamento de Mecánica Aplicada e Ingeniera de Proyectos, UCLM, angel.mromero@uclm.es

4 Dr. Ingeniero Agrónomo. Contratado Doctor; Departamento de Producción Vegetal y Tecnología Agraria, UCLM- ETSIAM; MiguelAngel.Moreno@uclm.es

${ }^{5}$ Dr. Ingeniero Agrónomo. Catedrático de UCLM. Centro Regional de Estudios del Agua. Albacete. jose.tarjuelo@uclm.es

\section{Resumen}

En zonas colectivas de riego, la tarifa a pagar por los agricultores está muy condicionada con el gasto energético de los equipos de bombeo, relacionada a su vez con la cantidad de agua aplicada a los cultivos. Así pues, es necesario el desarrollo de herramientas de ayuda a la toma de decisiones que busquen mejorar la eficiencia en el uso del agua y la energía, sin comprometer el rendimiento y productividad de los cultivos. El objetivo del trabajo es analizar el consumo energético en una estación de bombeo de una red colectiva de riego a la demanda ante distintos escenarios de riego en parcela y de presiones en la cabecera. La metodología propuesta ha sido aplicada a la zona regable de Tarazona de La Mancha (Albacete, España). Se han establecidos dos manejos de riego en parcela (con y sin déficit) para los principales cultivos de la zona y diferentes estrategias de presiones en la cabecera (variable y fija), para analizar el efecto sobre la eficiencia y consumo energético del bombeo. Para ello, se ha utilizado una herramienta de simulación desarrollada en MATLAB ${ }^{\circ}$, en combinación con el motor de cálculo EPANET ${ }^{\circledR}$, y se ha aplicado como ejemplo a un día de elevada demanda de agua en la red. Los resultados indican que la utilización de presión en cabecera variable y manejo deficitario, genera ahorros energéticos de 12,43\% en relación al mismo manejo con presión fija.

\section{1) Introducción. Objetivo del trabajo.}

En regiones semiáridas como Castilla-La Mancha (C-LM), el regadío en la agricultura permite diversificar los cultivos, garantizar un mayor nivel de producción y, en muchos casos, mantener la viabilidad económica de las explotaciones. En estas zonas, donde el principal factor limitante es la escasez de los recursos hídricos, junto con el hecho de tratarse en su mayoría de aguas subterráneas, hace que el coste ligado a la extracción y aplicación del agua con el riego sea uno de los factores más relevantes dentro de los costes de producción, debido al elevado coste de la energía.

El conocimiento preciso del posible ahorro de agua aplicada en parcela (mejorando la programación de riegos, utilizando riego deficitario, etc.) y de energía que se puede conseguir con una adecuada gestión de la red, resulta de gran interés por su correspondiente repercusión económica y medioambiental. Este aspecto tiene mayor 
importancia en grandes redes colectivas de riego, donde el uso colectivo de los recursos debe optimizarse para garantizar la sostenibilidad del regadío.

En la gestión de estas redes se plantean alternativas de manejo a turnos o a la demanda, si bien en ambos casos cada agricultor realiza su programación del riego en parcela, utilizando criterios no siempre optimizados para cada uno de sus cultivos.

Por lo tanto, para mejorar la gestión de una red colectiva de riego se hace necesario profundizar en el manejo del riego en parcela, buscando estrategias que permitan gestionar el agua de riego de modo óptimo para cada cultivo. En condiciones de escasez de recursos hídricos, con regadíos infradotados, o en las que los costes energéticos de extracción de aguas subterráneas son muy altos, la aplicación de técnicas tales como el Riego Deficitario Controlado (RDC) (English, 1990) permiten reducir costes productivos, e incrementar la eficiencia en el uso del agua por parte de los cultivos (López-Urrea et al., 2009), consiguiendo un mayor rendimiento por unidad de volumen de agua aplicada al cultivo a costa de reducir el rendimiento por unidad de superficie. Esta técnica consiste en reducir el aporte de agua durante las etapas menos sensibles al estrés hídrico para evitar o limitar el déficit en las más sensibles.

El objetivo del trabajo es analizar el consumo energético en una estación de bombeo de una red colectiva de riego a la demanda, considerando dos manejos de la presión en cabecera, fija o variable, y dos manejos del riego en parcela, con riego deficitario controlado y sin él.

\section{2) Material y métodos}

\subsection{Caso de estudio}

La metodología propuesta ha sido aplicada en una Comunidad de Regantes (CCRR) en Tarazona de La Mancha (Albacete, España). La zona regable de 1044 ha, está compuesta por dos redes colectivas de riego a la demanda independientes (Sector I y Sector II).

En el sector de estudio (sector I), el agua se distribuye mediante un grupo de rebombeo compuesto por 10 bombas iguales (140 CV por bomba) conectadas en paralelo, dos de ellas con variador de velocidad (Tabla 1). La estación de bombeo funciona con regulación manométrica en cabecera, con presión fija de $52 \mathrm{~m}$.

Tabla 1. Características hidráulicas de los dos sectores de SORETA.

\begin{tabular}{|c|c|}
\hline Características & Sector I \\
\hline Superficie regable (ha) & 550,2 \\
\hline Numero de hidrantes & 389 \\
\hline Volumen del embalse $\left(\mathrm{m}^{3}\right)$ & 23000 \\
\hline Número de sondeos & 4 \\
\hline Numero de bombas para el rebombeo & 10 \\
\hline Potencia de las bombas para el rebombeo (CV) & 140 \\
\hline Longitud total de tuberías $(\mathrm{m})$ & 30504 \\
\hline
\end{tabular}

Este sector tiene una superficie regable de 550,2 ha, dedicadas principalmente a cultivos herbáceos (cereales de invierno y verano, cebolla y ajo), y con predominio de sistema de riego mediante cobertura total enterrada. La presencia de cultivos leñosos es menor (aproximadamente del 20\%), aunque ha aumentado en los últimos años, con la transformación de los sistemas de riego en parcela por aspersión a sistemas de riego localizado.

\subsection{Metodología propuesta}


En la Figura 1, se muestra el diagrama de flujo de los escenarios de manejo (con y sin déficit) y de presión en cabecera (variable y fija), que se pretenden comparar en una red colectiva de riego ya calibrada en trabajo previos (Moreno et al, 2007a). La comparación de resultados se realizará evaluando el consumo energético de la red para un día de elevada demanda hídrica, utilizando para ello una herramienta programada en MATLAB® en combinación con el motor de cálculo EPANET® (Rossman, 2001).

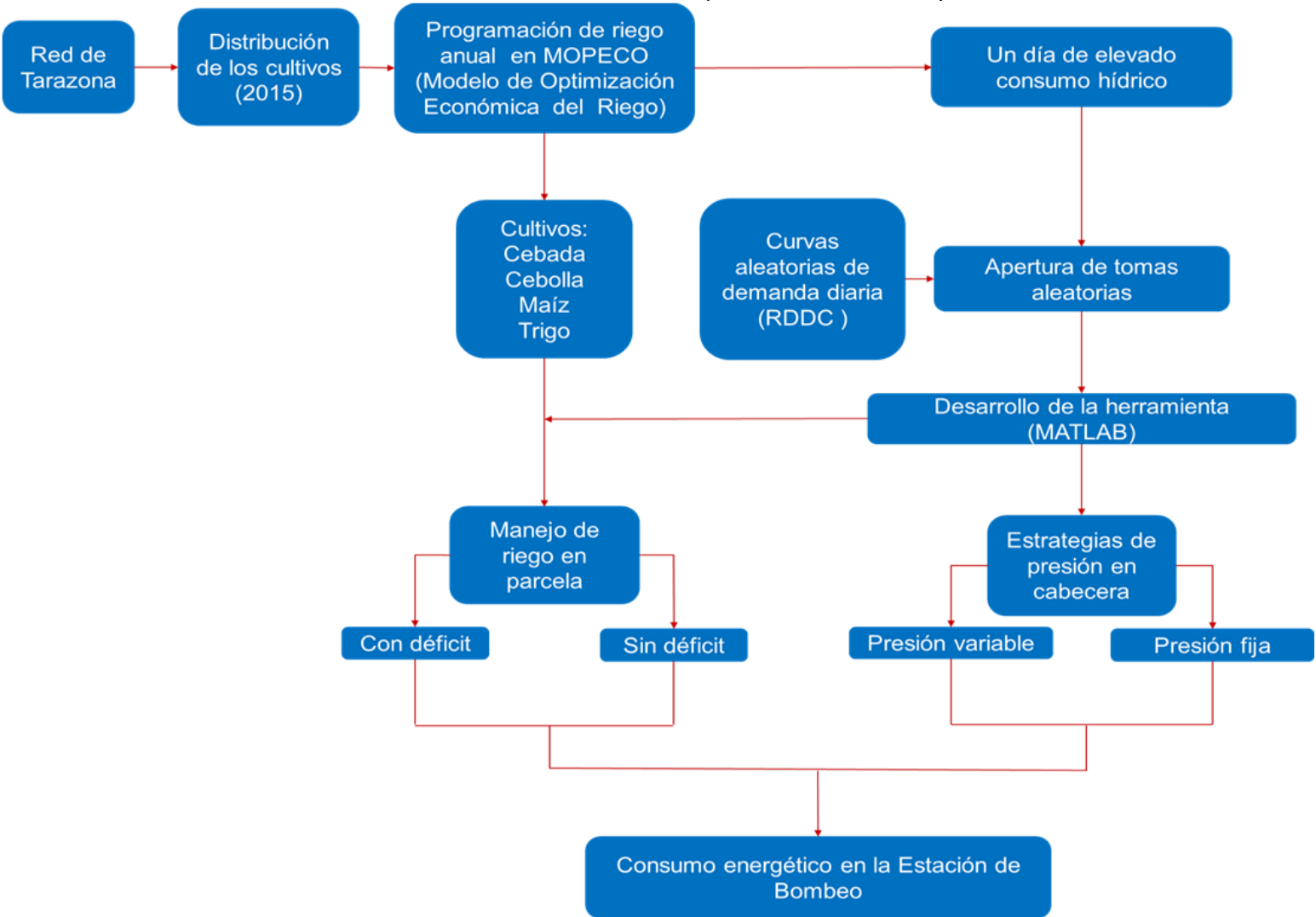

Figura 1. Diagrama de flujo de la metodología aplicada.

Como punto de partida, se ha utilizado la distribución de los cultivos para el sector de estudio en el año de 2015, donde aproximadamente la mitad de la superficie está ocupada por los cultivos de cebada (30\%), trigo (9\%), maíz (6\%) y cebolla (4\%) (Fig. 2), representando un consumo de agua anual del $56 \%$ del que se presenta en la red.

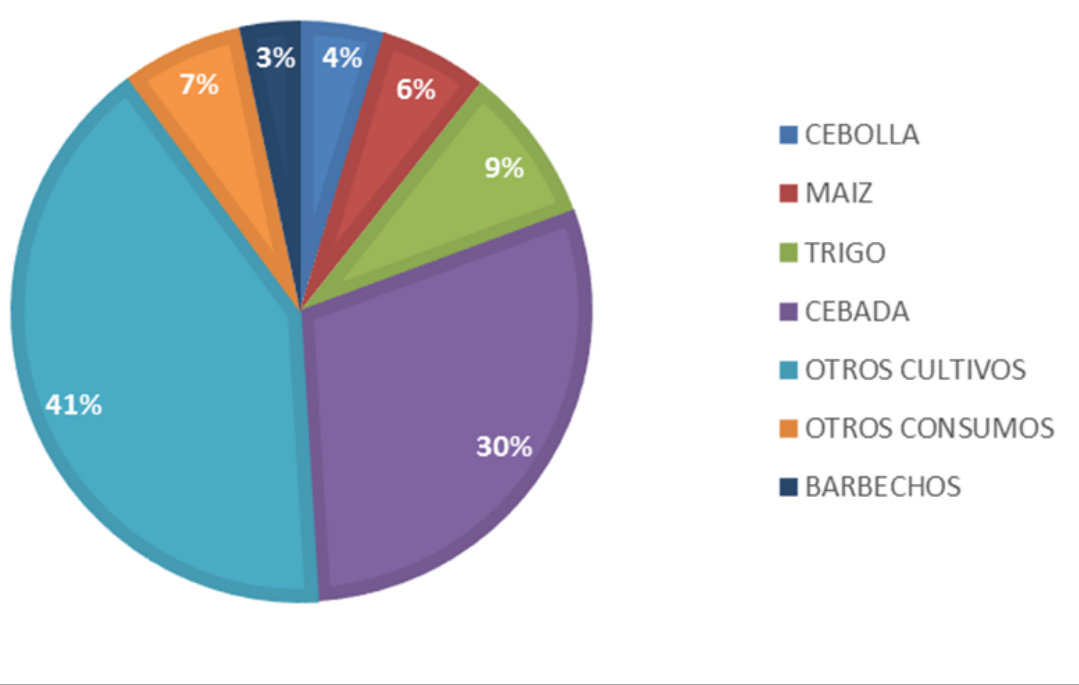

Figura 2. Distribución de los cultivos (\%) para el año 2015. 
Para estos cultivos, se han planificado las programaciones de riego optimizadas para el año 2015, con dos manejos de riego en parcela, uno con riego deficitario, con el objetivo de conseguir los niveles de déficit recogidos en la Tabla 3 durante el ciclo de cultivo, y otro sin riego deficitario. La programación de ambos tipos de riegos se han realizado utilizando el método de balance simplificado diario de agua en el suelo usando el módulo destinado para este fin en el programa MOPECO (Modelo de Optimización Económica del Riego) (Domínguez, et al., 2012ab) que aplica la metodología FAO (Allen et al., 1998), y que ha sido previamente calibrado para los cultivos seleccionados.

En la Tabla 2, se muestra la duración en días y los valores de los coeficientes de cultivo (kc), para cada etapa de desarrollo de los mismos, utilizados para establecer las programaciones de riego.

Tabla 2. Duración de las etapas y valores de coeficiente de los cultivos (kc).

\begin{tabular}{|l|c|c|c|c|c|c|c|c|}
\hline & \multicolumn{2}{|c|}{ Cebada } & \multicolumn{2}{c|}{ Cebolla } & \multicolumn{2}{c|}{ Maíz } & \multicolumn{2}{c|}{ Trigo } \\
\hline $\begin{array}{l}\text { Etapa } \\
\text { fenológica }\end{array}$ & Días* & $\mathrm{kc} *$ & Días* & $\mathrm{kc} *$ & Días* & $\mathrm{kc} *$ & Días* & $\mathrm{kc} c^{\star \star}$ \\
\hline Establecimiento & 102 & $0,30-0,30$ & 60 & $0,65-0,65$ & 41 & $0,30-0,30$ & 98 & $0,30-0,30$ \\
\hline $\begin{array}{l}\text { Desarrollo } \\
\text { vegetativo }\end{array}$ & 45 & $0,30-1,15$ & 31 & $0,65-1,20$ & 39 & $0,30-1,10$ & 40 & $0,30-1,15$ \\
\hline Etapa media & 16 & $1,15-1,15$ & 47 & $1,20-1,20$ & 38 & $1,10-1,10$ & 36 & $1,15-1,15$ \\
\hline Maduración & 20 & $1,15-0,45$ & 19 & $1,20-0,75$ & 27 & $1,10-0,55$ & 21 & $1,15-0,45$ \\
\hline
\end{tabular}

*Días: Duración en días de cada etapa fenológica; ${ }^{* \star k c: ~ C o e f i c i e n t e ~ d e l ~ c u l t i v o . ~}$

Para el manejo del Riego Deficitario Controlado (RDC) se ha determinado el nivel de déficit que debe aplicarse en cada etapa del cultivo para lograr el máximo rendimiento posible bajo un cierto nivel de déficit global objetivo. El nivel de déficit global para los cultivos se ha establecido en base al manejo común en la zona (Tabla 3), con reducciones de entre el $20 \%$ y el $30 \%$ sobre los consumos máximos. La distribución del déficit hídrico por etapas en los cultivos se realizó siguiendo las recomendaciones de Domínguez et al (2012ab) (Tabla 3), aplicando la técnica de Riego Deficitario Optimizado por Etapas (ORDI- Optimized Regulated Deficit Irrigation), que determina los niveles de déficit óptimos a aplicar (relación $\mathrm{ET}_{\mathrm{a}} / \mathrm{ET}_{\mathrm{m}}$ ) en cada etapa de desarrollo de los cultivos herbáceos en función del nivel de déficit global que se desee alcanzar.

Para los distintos escenarios de manejo, se han seleccionado dos parcelas de seguimiento de cada cultivo, en las que previamente se han evaluado los sistemas de riego para definir las condiciones de funcionamiento (presión de funcionamiento, caudales descargados y uniformidad de distribución del agua). Además se ha instalado un transductor de presión en el aspersor que presentaba aproximadamente la presión media en cada parcela de seguimiento, lo que ha permitido registrar cada dos minutos la presión de funcionamiento, determinando factores como los momentos de inicio y finalización de los riegos, presiones medias de trabajo y el agua aplicada al cultivo en cada riego.

Para definir las simulaciones de funcionamiento de la red, se ha escogido una semana (entre el 11 y el 17 de mayo) donde se dan las condiciones de mayor demanda de agua en la red (Fig. 3), dado que en este período se encuentran implantados cultivos de invierno (cereales) y de verano (cebolla y maíz), siendo los primeros los que demandan una mayor proporción del agua de riego en el conjunto de la red. 


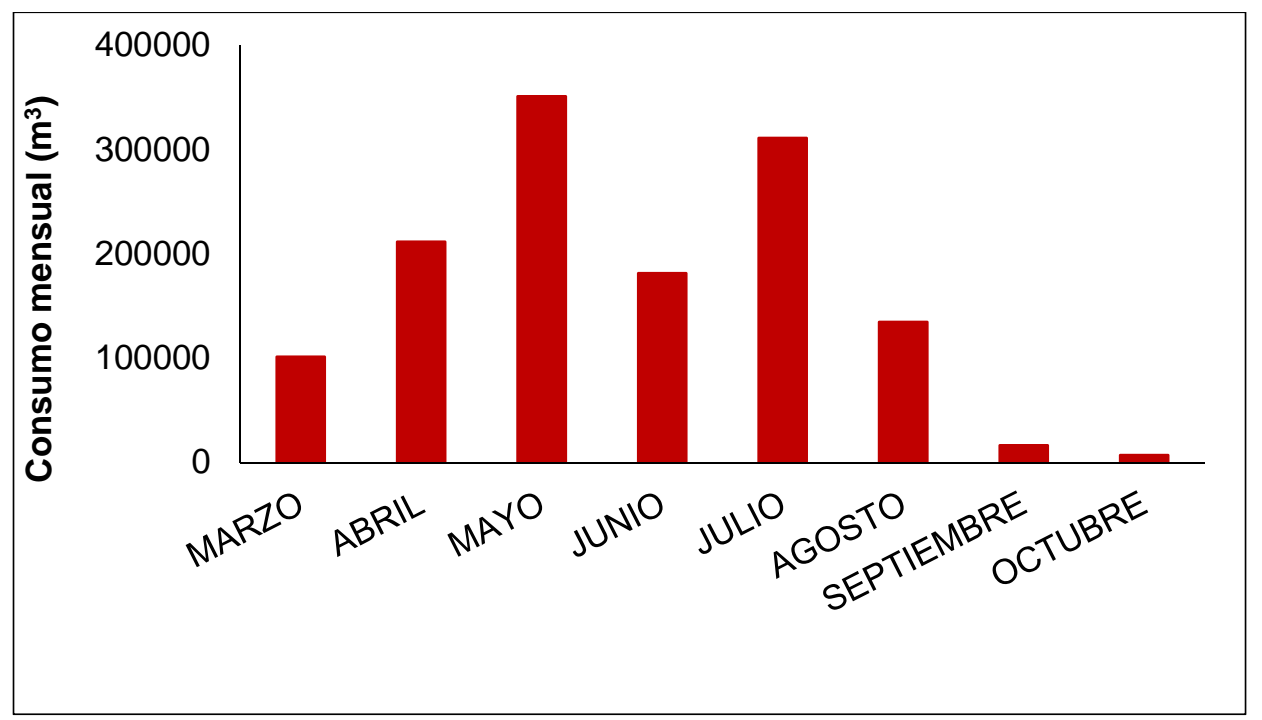

Figura 3. Consumo mensual de agua en la CCRR de Tarazona para el año de 2015.

Para realizar las simulaciones, se ha desarrollado una herramienta en MATLAB $®$ en combinación con el motor de cálculo EPANET® (Rossman, 2001). Con esta herramienta se determinarán los caudales circulantes por las líneas de la red así como las presiones, permitiendo así obtener la eficiencia energética de la estación de bombeo y su consumo energético para los escenarios planteados.

Para el desarrollo de las simulaciones, se parte de una distribución de apertura de tomas al azar, basada en apertura aleatoria utilizando metodología de Curvas Aleatorias de Demanda Diaria (RDDC) (Moreno et al. 2007a), que tiene en cuenta la probabilidad de apertura de tomas para un día; el tiempo de riego ( $\mathrm{Tr}$, en horas); el número de sectores; la Jornada Efectiva de Riego (horas); y el Intervalo de riegos (días). Además, el tiempo de inicio de apertura también se ha generado de forma aleatoria.

En las simulaciones realizadas, el número de tomas abiertas durante un día son las mismas para los dos manejos (con y sin déficit), variando únicamente el $\mathrm{Tr}$ en cada caso (Tabla 3).

Tabla 3. Determinación de tiempo de riego para una semana.

\begin{tabular}{|c|c|c|c|c|c|c|c|c|}
\hline \multirow{2}{*}{ Cultivo } & \multirow{2}{*}{$\begin{array}{c}\text { Etapa } \\
\text { fenológica }\end{array}$} & \multirow{2}{*}{$\begin{array}{c}\mathrm{ETm}^{\star} \\
(\mathrm{mm} \\
\left.\text { semana }^{-1}\right)\end{array}$} & \multirow{2}{*}{$\begin{array}{c}\text { Intervalo } \\
\text { de riego } \\
\text { (días) }\end{array}$} & \multirow{2}{*}{$\begin{array}{l}\text { Riegos } \\
\text { por } \\
\text { semana }\end{array}$} & \multicolumn{2}{|c|}{ Déficit } & \multicolumn{2}{|c|}{$\begin{array}{l}\text { Tiempo de } \\
\text { riego }(\mathrm{h})\end{array}$} \\
\hline & & & & & Global & Etapa & $\begin{array}{c}\text { Sin } \\
\text { déficit }\end{array}$ & $\begin{array}{l}\text { Con } \\
\text { déficit }\end{array}$ \\
\hline Cebada & Etapa media & 53,02 & 3,5 & 2 & 0,70 & 0,75 & 4,5 & 3,5 \\
\hline Cebolla & $\begin{array}{l}\text { Desarrollo } \\
\text { vegetativo }\end{array}$ & 34,15 & 3,5 & 2 & 0,80 & 0,63 & 3,0 & 2,0 \\
\hline Maíz & Establecimiento & 13,83 & 7,0 & 1 & 0,80 & 0,80 & 2,5 & 2,0 \\
\hline Trigo & Etapa media & 53,02 & 3,5 & 2 & 0,75 & 0,85 & 4,5 & 4,0 \\
\hline
\end{tabular}

*Etm: Evapotranspiración máxima semanal de cultivo $(E t m=$ Evapotranspiración $x$ Coeficiente de cultivo $(\mathrm{kc})$.

La jornada efectiva de riego (JER) ha sido de 24 horas, referente a un día de fin de semana, correspondiendo al período de tarifa más barata contratada por la CCRR.

Con las tomas seleccionadas y los tiempos de riego en cada parcela para cada tipo manejo de riego (deficitario y no deficitario), se han determinado los caudales circulantes en las tuberías así como las presiones en cada toma de la red, tanto para el caso de la presión variable necesaria en cabecera capaz de garantizar una presión mínima de 35 m.c.a en el hidrante abierto más desfavorable, como para el caso de considerar el valor de presión fija en cabecera empleado en la regulación actual de la CCRR (52 m.c.a). 
Para analizar el consumo de energía $\left(\mathrm{kW} \mathrm{h}^{-1}\right)$ de la estación de bombeo en las distintas condiciones de presión y caudal, se ha determinado la eficiencia de la estación de bombeo para distintos rangos de caudal y presión en cabecera empleando el Modelo de Análisis de Eficiencia Energética en Estaciones de Bombeo (MAEEB) (Moreno et al. 2007b).

Para determinar la eficiencia actual de la estación de bombeo, ha sido necesario adquisición de datos en la campaña 2015, relativos a parámetros hidráulicos y eléctricos en la estación de bombeo. Los parámetros eléctricos se han determinado con un analizador de redes QNA (Circutor, Barcelona, España) mediante un registro continuo de los parámetros eléctricos (intensidad, voltaje, potencia, etc). Es un equipo especialmente diseñado para el análisis de la calidad de suministro eléctrico, que permite detectar cualquier evento que se produzca en la red (huecos, interrupciones, armónicos, potencia, frecuencia, etc.) (Moreno, 2005; QNA, 2006, Córcoles 2009). El QNA se instaló entre el transformador de media a baja tensión y a la entrada a la estación de bombeo, con registro de medidas cada diez minutos, desde el inicio de la campaña de riegos.

El registro de presión se ha realizado de forma continua mediante un transductor de presión (Druck, Leicester, UK) con un registrador de datos (Dickson, modelo ES-120). Este dispositivo, con un rango de medida de 0 a 6 bar, se instaló en el colector de impulsión de la estación de bombeo, siendo alimentado por una batería de $12 \mathrm{~V}$.

Además, se realizó una evaluación de las bombas de la EB (mediciones de caudal, presión y potencia absorbida) determinando el caudal descargado por cada una de las bombas, utilizando un caudalimetro de ultrasonidos (PT-868, de Panametric) así como su consumo eléctrico mediante un analizador de redes tipo AR5 (Circutor, Barcelona, España).

\section{3) Resultados y discusiones}

Se han realizado numerosas simulaciones para comprobar el funcionamiento de la herramienta, mostrando una de ellas para describir los resultados. En la Figura 4 se muestra la similitud de distribución de los caudales simulados por la herramienta, frente a los datos reales de caudal medidos en la campaña 2015.

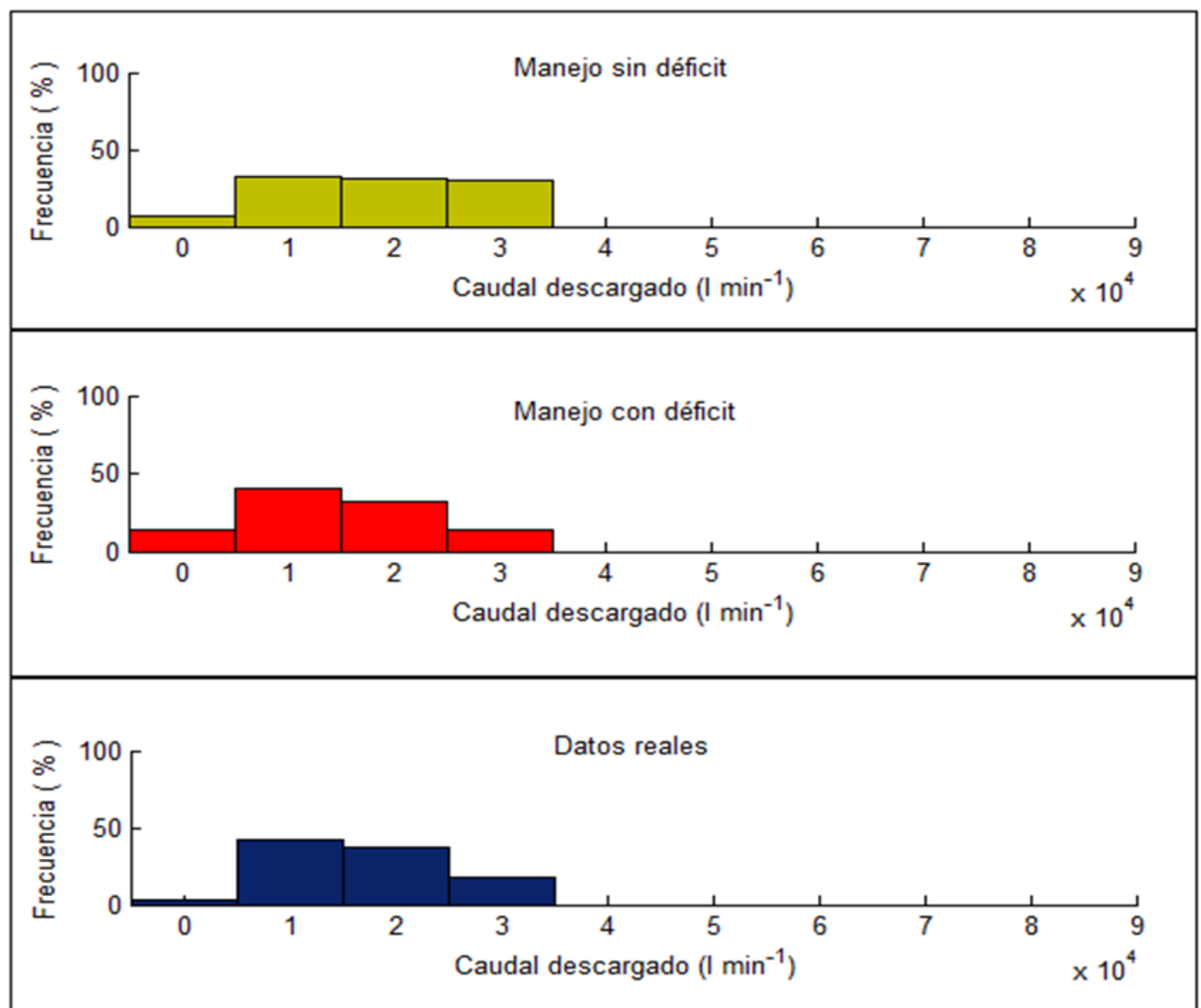

Figura 4. Frecuencia de caudales simulados y real. 
En el caso del manejo sin déficit, la Figura 4 muestra que el 93\% de los caudales demandados se concentran en un rango que varían de 5000 a $35000 \mathrm{I} \mathrm{min}^{-1}$. La frecuencia de caudales en el manejo con déficit es semejante a la distribución de frecuencias de caudal de los datos reales. Para este manejo, los caudales más bajos (entre 0 y $15000 \mid \mathrm{min}^{-1}$ ), representan más de la mitad $(54,17 \%)$ de los caudales demandados.

Para esta simulación, la probabilidad de tomas abiertas, generada por medio de las curvas aleatoria de demanda (hecha en Matlab ${ }^{\circledR}$ ), ha establecido al azar un total de 36 tomas abiertas, utilizada para los dos manejos de riego. En el caso de los datos reales, no se dispone de información relativa al número de tomas abiertas en un determinado momento ni tampoco el número total de parcelas que han regado en un día. En relación con esto, en base a un día de elevada demanda en la red, con los datos medidos en la estación de bombeo ha sido posible determinar la distribución de los caudales demandados en la red, de utilidad para poder validar las simulaciones realizadas con la herramienta. De este modo, la apertura aleatoria de tomas intenta representar un escenario de riego habitual en la zona, representando así una petición real de puesta en riego por parte de los agricultores.

En la Figura 5 se muestra la distribución de los caudales para los escenarios planteados (riego sin déficit y con déficit) a partir de la aleatoriedad de aperturas de tomas.

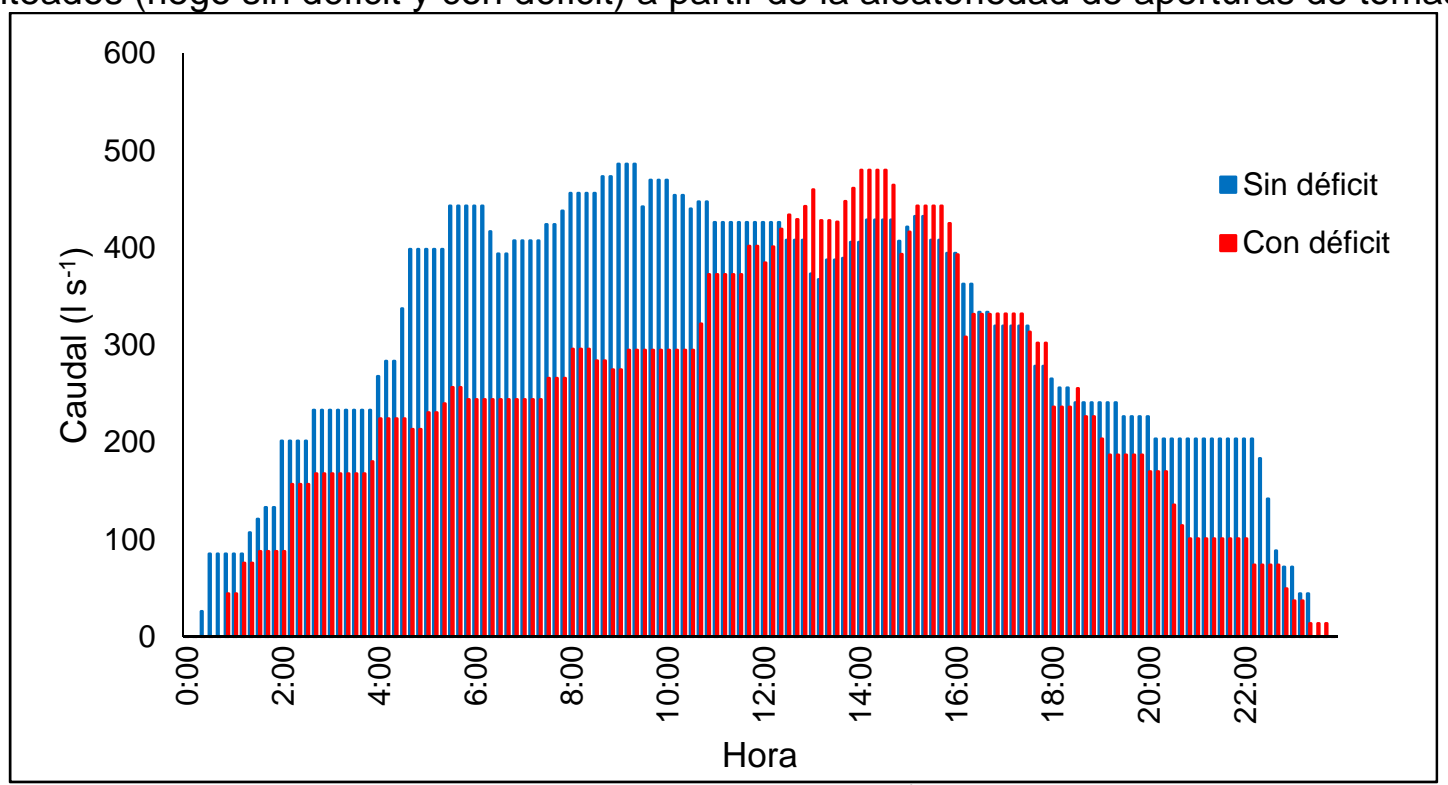

Figura 5. Distribución de los caudales $\left(\mathrm{I} \mathrm{S}^{-1}\right)$ para la simulación.

Para los dos manejos (sin y con déficit), la distribución de caudales muestra una distribución homogénea, con mayores demandas a lo largo del día para el manejo sin déficit. Las mayores demandas de caudales para el manejo sin déficit, se debe al mayor tiempo necesario de riego para los cultivos, además, la distribución de los dos manejos son distintos, no presentando la misma forma a lo largo del día, porque, a pesar de utilizar las mismas tomas, el momento de inicio de su apertura es distinto.

Teniendo en cuenta el volumen de agua total aportado para el periodo considerado, cabe destacar que, en el caso de riego con déficit, el volumen ha sido inferior (alrededor de 21\%), en comparación con el manejo sin déficit.

Utilizando la herramienta desarrollada, la presión en cabecera para los dos manejos, alcanza valores medios (alrededor de $40 \mathrm{~m}$ ) inferiores al sistema de regulación actual de la estación de bombeo (52 m) (Tabla 4).

Tabla 4. Presiones en cabecera simulados para los dos manejos de riego.

\begin{tabular}{|c|c|c|c|c|}
\hline Escenario & Media & Mínimo & Máximo & Desviación estándar \\
\hline Sin déficit & 41,50 & 38,10 & 45,17 & 2,69 \\
\hline Con déficit & 40,62 & 38,10 & 43,86 & 2,09 \\
\hline
\end{tabular}


En relación a los rendimientos de la estación de bombeo, aunque las diferencias son pequeñas, los valores obtenidos fueron mayores para la presión fija en cabecera que para la variable, siendo algo mayor cuando se ha simulado para un manejo sin déficit en ambos casos (Tabla 5).

Tabla 5. Rendimiento medio de la EB para presión variable y fija.

\begin{tabular}{|l|c|c|c|c|}
\hline \multirow{2}{*}{} & \multicolumn{2}{|c|}{ Presión variable } & \multicolumn{2}{c|}{ Presión fija } \\
\cline { 2 - 5 } & Sin déficit & Con déficit & Sin déficit & Con déficit \\
\hline Rendimiento (\%) & 54,53 & 53,54 & 59,46 & 59,00 \\
\hline
\end{tabular}

El consumo de energía por la estación de bombeo (Fig. 6A y B) mostró una evolución similar a la obtenida con la distribución de caudales (Fig. 5). El consumo energético a lo largo del día, es mayor cuando se ha utilizado la presión fija, independiente del tipo de manejo de riego adoptado.

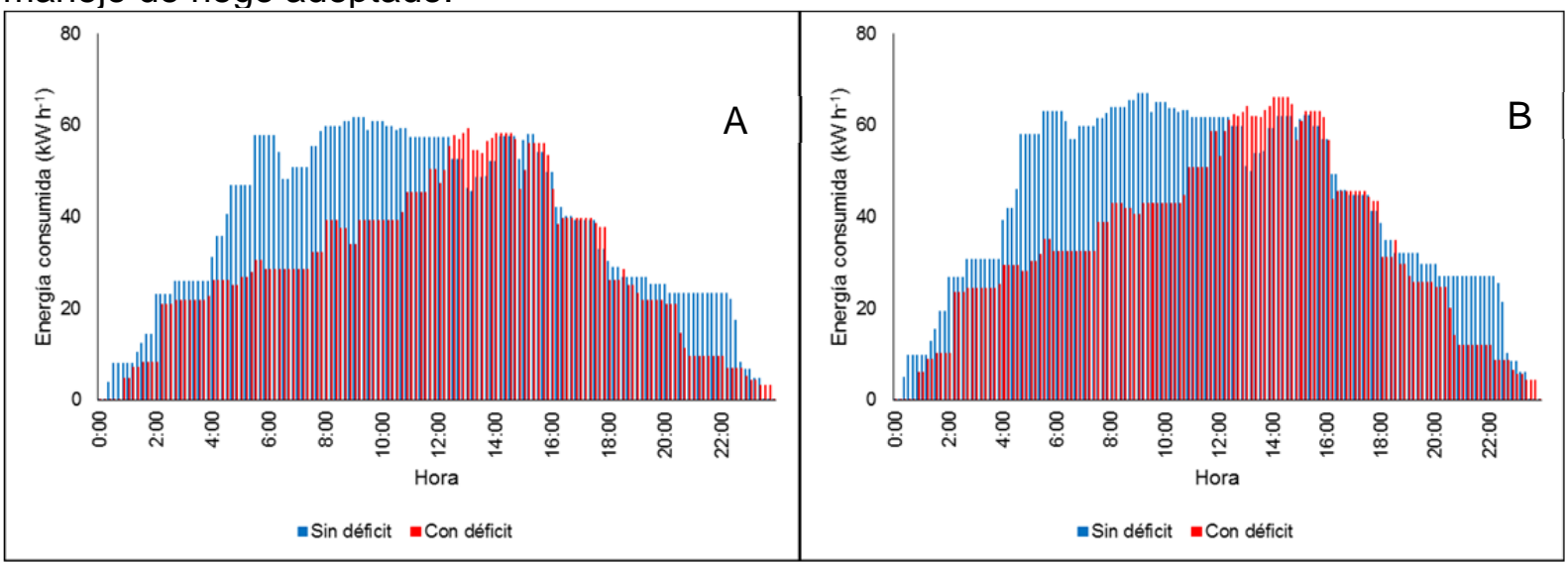

Figura 6. Consumo energético para la EB para la presión variable (A) y fija (B) para un día de simulación.

En la Tabla 6 se muestran los valores de consumo energético total para un día simulado. Es posible observar que en todos los casos analizados, el consumo fue menor que el consumo real obtenido por la red, excepto cuando se ha utilizado una presión fija con manejo sin déficit, que presentan un consumo energético superior $(3,33 \%)$ al medido en la EB.

Tabla 6. Consumo energético total para un día simulado.

\begin{tabular}{|l|c|c|c|c|c|}
\hline & \multicolumn{2}{|c|}{ Presión variable } & \multicolumn{2}{c|}{ Presión fija } & \multirow{2}{*}{ Datos Reales } \\
\cline { 2 - 5 } & Sin déficit & Con déficit & Sin déficit & Con déficit & \\
\hline Energía consumida $\left(\mathrm{kW} \mathrm{h}^{-1}\right)$ & 5509,59 & 4274,49 & 6213,43 & 4881,22 & 6012,93 \\
\hline
\end{tabular}

Entre los escenarios planteados, la presión variable y el manejo de riego deficitario en parcela, ha conseguido el mayor ahorro energético (Tabla 7). En comparación con los datos reales, con ese escenario se consigue un ahorro de $28,91 \%$ y cuando se compara con el mismo manejo pero adoptando la estrategia de mantener una presión fija, se ha conseguido un ahorro de $12,43 \%$.

\section{4) Conclusiones}

El presente trabajo muestra la utilidad de una herramienta generada para analizar la influencia que tiene las programaciones de riego en parcela sobre el consumo energético y el manejo de las estaciones de bombeo. De este modo se puede analizar el comportamiento de la estación de bombeo y de la red en estas zonas a través del conocimiento de los escenarios de demanda derivados de las programaciones del riego en parcela. Por todo ello, 
es un aspecto esencial integrar la programación de riego en parcela como una actividad complementaria dentro de las actividades realizadas por los gestores de las redes de riego.

El uso de manejos de riego en parcela y estrategia de presión en cabecera conllevan un ahorro en el consumo energético y, consecuentemente, económicos, cuando se utiliza manejo de riego deficitario en parcela y presiones variables en la cabecera.

Se pretende que la herramienta generada pueda servir para la optimización de recursos hídricos y energéticos en redes colectivas, a partir de programaciones de riego en parcela. De esta forma, se continuará con el desarrollo de la herramienta para profundizar el comportamiento utilizando diferentes días de la semana, así como etapa de mínima y máxima demanda de la red.

\section{5) Bibliografía}

Allen, R.G. Pereira, L.S. Raes, D. Smith,M. 1998. Crop evapotranspiration: guidelines for computing crop water requirements. FAO. Irrigation and Drainage Paper, 56, 297.

Córcoles, J.I. 2009. La gestión del agua y la energía en el regadío mediante técnicas de "Benchmarking". Tesis Doctoral, escuela Técnica Superior de Ingenieros Agrónomos, Universidad Castilla la Mancha, Albacete, España.

Domínguez, A, Juan, J,A, Tarjuelo, J,M, Martínez, R,S, Martínez-Romero, A, (2012a), Determination of optimal regulated deficit irrigation strategies for maize in a semi-arid environment, Agricultural Water Management, 110, 67-77.

Domínguez, A, Jiménez, M, Tarjuelo, J,M, Juana, J,A, Martínez-Romero A, Leite, K,N, (2012b), Simulation of onion crop behavior under optimized regulated deficit irrigation using MOPECO model in a semi-arid environment, Agricultural Water Management, $113,64-75$.

English, M.J, (1990), Deficit irrigation. I: Analytical framework. Journal of Irrigation and Drainage Engineering, 116, 399-412.

López-Urrea, R, Montoro, A, González-Piqueras, J, López-Fuster, P, Fereres, E. (2009). Water use of spring wheat to raise water productivity. Agric. Water Manage., $96,1305-1310$.

Moreno, M. A., Planells, P., Ortega, J. F., Tarjuelo, J. (2007a). New methodology to evaluate flow rates in on-demand irrigation networks. Journal of Irrigation and Drainage Engineering, 133(4), 298-306.

Moreno, M. A., Carrión, P. A., Planells, P., Ortega, J. F., Tarjuelo, J. M. (2007b). Measurement and improvement of the energy efficiency at pumping stations. Biosystems Engineering, 98(4), 479-486.

Rossman, L, A, (2001), EPANET 2, Users Manual, Water supply and Water Resources Division National Risk Management Research Laboratory, Cincinati, USA: U,S, Enviromental Protection Agency. 